\title{
ON FIXED POINTS OF THE COMPOSITE OF COMMUTING FUNCTIONS
}

GLEN BAXTER ${ }^{1}$

1. Introduction. Let $f(t)$ and $g(t)$ be continuous functions mapping $[0,1]$ into $[0,1]$ which commute under substitution, i.e., $f(g(t))$ $=g(f(t))$. In 1954, E. Dyer "conjectured" 2 that $f(t)$ and $g(t)$ must have a common fixed point in $[0,1], t_{0}=f\left(t_{0}\right)=g\left(t_{0}\right)$ for some $t_{0}$ in $[0,1]$. For certain special functions $f(t)$ and $g(t)$ the conjecture has been verified, but in general the question of existence of this common fixed point remains open.

An equivalent way of phrasing the conjecture is to say that $f(t)$ and $h(t)=f(g(t))$ must have a common fixed point. In this connection, it is known that $f(t)$ and $g(t)$ "permute" the fixed points of $h(t)$. In particular, if $h(t)$ has a finite number of fixed points $t_{1}, \cdots, t_{n}$, then $f\left(t_{1}\right), \cdots, f\left(t_{n}\right)$ is a permutation of $t_{1}, \cdots, t_{n}$. (This follows from the fact that (1) $f(t)$ and $h(t)$ commute and (2) $f\left(t_{i}\right)=f\left(t_{j}\right)$ implies $t_{i}=t_{j}$ by applying $g(\cdot)$ to both sides.) In this note, we investigate more closely the permutation just mentioned for the case in which $h(t)$ has a finite number of fixed points.

We begin with the observation that any fixed point $t_{0}$ of $h(t)$ is one of three types. Type I, up-crossing: $h(t)$ passes from below to above the diagonal as $t$ increases through $t_{0}$. Type II, down-crossing: $h(t)$ passes from above to below the diagonal as $t$ increases through $t_{0}$. Type III, touching: $h(t)$ does not cross the diagonal at $t_{0}$. Included in Type II will be (1) $t=1$ if $h(1)=1$ and $h(t)>t$ near $t=1$, and (2) $t=0$ if $h(0)=0$ and $h(t)<t$ near $t=0$. Included in Type III will be (1) $t=1$ if $h(1)=1$ and $h(t)<t$ near $t=1$, and (2) $t=0$ if $h(0)=0$ and $h(t)>t$ near $t=0$. We now state our main result, which indicates how $f(t)$ and $g(t)$ "preserve" the local behavior of $h(t)$ at fixed points.

Theorem. Let $h(t)$ have a finite number of fixed points. Then, $f(t)$ and $g(t)$ permute the fixed points of each type.

We can use the theorem to obtain some information about Dyer's

Presented to the Society, February 18, 1963; received by the editors March 13, 1963 and, in revised form, July 3, 1963.

1 This work was supported in part by the United States Air Force through the Air Force Office of Scientific Research of the Air Research and Development Command.

${ }^{2}$ Dyer raised the question in 1954. Shields asked the same question in 1955, and Dubins asked it in 1956. Isbell raised a more general question in 1957 [1]. 
conjecture. For any function $h(t)$, there must be one more downcrossing than up-crossing. If $h(t)$ has fewer than five fixed points, there is only one up-crossing or only one down-crossing. In either case, the permutation $\left\{f\left(t_{i}\right)\right\}$ has a fixed point, verifying the conjecture: The theorem gives useful information in many cases, but it gives no information about the conjecture even if $h(t)$ has only five fixed points, two up-crossings and three down-crossings.

We wish to express our thanks to Professor J. Joichi for providing some interesting and useful comments on our results.

2. Proof of the theorem. Let $0 \leqq t_{1}<t_{2} \cdots<t_{n} \leqq 1$ denote the fixed points of $h(t)=f(g(t))$, and let $I_{k}$ be the interval $t_{k} \leqq t \leqq t_{k+1}$. For any interval $I$, let $f(I)$ denote the interval into which $f(t)$ maps $I$. If $h(t) \geqq t$ on $I_{k}$, we say $I_{k}$ is an up-interval; if $h(t) \leqq t$ on $I_{k}$, we say $I_{k}$ is a down-interval. If two intervals $I_{k}$ and $I_{m}$ are both up-intervals or both down-intervals, we say they are alike. From now on, $f(g(t))$ will be denoted by simply $f g(t)$.

We establish two auxiliary lemmas.

LeMma 1. Let $f\left(t_{m}\right)$ belong to $f\left(I_{k}\right)$ for some $t_{m}$. Then, $t_{m} \geqq t_{k}$ if $I_{k}$ is an $u p$-interval, and $t_{m} \leqq t_{k+1}$ if $I_{k}$ is a down-interval.

For the proof, let $I_{k}$ be an up-interval and let $a$ be a point in $I_{k}$ such that $f\left(t_{m}\right)=f(a)$. Then, $t_{m}=g f\left(t_{m}\right)=g f(a) \geqq a$. The other half of the lemma is proved in a similar manner. Note, in particular, that if $f\left(t_{k}\right)$ and $f\left(t_{k+1}\right)$ are not successive fixed points, then there is at least one $t_{m} \neq t_{k}, t_{k+1}$ for which $f\left(t_{m}\right)$ belongs to $f\left(I_{k}\right)$. The direction of the inequality $h(t) \leqq t$ or $h(t) \geqq t$ determines the location of $t_{m}$ with respect to $I_{k}$. The next lemma considers the case in which $f\left(t_{k}\right)$ and $f\left(t_{k+1}\right)$ are successive fixed points.

LEMMA 2. Let $f\left(t_{k}\right)$ and $f\left(t_{k+1}\right)$ be successive fixed points, say $t_{m}$ and $t_{m+1}$. If $f\left(t_{k}\right)=t_{m}$, then $I_{k}$ and $I_{m}$ are alike. If $f\left(t_{k}\right)=t_{m+1}$, then $I_{k}$ and $I_{m}$ are not alike.

To prove the first part of the lemma, we must eliminate the case in which $I_{k}$ and $I_{m}$ are not alike. Since $\left\{f\left(t_{i}\right)\right\}$ and $\left\{g\left(t_{i}\right)\right\}$ are inverse permutations, we can assume without loss of generality that $I_{k}$ is an up-interval and $I_{m}$ is a down-interval. First, we show that

$$
f\left(I_{k}\right)=I_{m} \text { and } g\left(I_{m}\right)=I_{k} \text {. }
$$

For suppose there were $a_{0}$ in the open interval $\left(t_{k}, t_{k+1}\right)$ such that $f\left(a_{0}\right)=t_{m}$. Then, $a_{0}<g f\left(a_{0}\right)=g\left(t_{m}\right)=t_{k}$, a contradiction. Thus, $f(a)>t_{m}$ for all $a$ in $\left(t_{k}, t_{k+1}\right)$. On the other hand, if there were $a_{0}$ in the open 
interval $\left(t_{k}, t_{k+1}\right)$ such that $f\left(a_{0}\right)=t_{m+1}$, then there would exist $b_{0}$ in the interval $\left(t_{m}, t_{m+1}\right)$ with $g\left(b_{0}\right)=a_{0}$, and $b_{0}>f g\left(b_{0}\right)=f\left(a_{0}\right)=t_{m+1}$, a contradiction. This means that $f\left(I_{k}\right)=I_{m}$ and similarly $g\left(I_{m}\right)=I_{k}$. Next, we take any $a_{0}$ in the open interval $\left(t_{k}, t_{k+1}\right)$ and define

$$
b_{0}=f\left(a_{0}\right), \quad a_{n+1}=g\left(b_{n}\right), \quad b_{n+1}=f\left(a_{n+1}\right) .
$$

According to (1) and to the relations $a_{n+1}=g f\left(a_{n}\right)>a_{n}, b_{n+1}=f g\left(b_{n}\right)$ $<b_{n}$, it follows that $\left\{a_{n}\right\}$ is an increasing sequence of points in $I_{k}$ approaching $t_{k+1}$ and that $\left\{b_{n}\right\}$ is a decreasing sequence of points in $I_{m}$ approaching $t_{m}$. By (2), we are led to the contradiction

$$
t_{k+1}=\lim _{n \rightarrow \infty} a_{n+1}=\lim _{n \rightarrow \infty} g\left(b_{n}\right)=g\left(t_{m}\right)=t_{k} .
$$

The proof of the second half of the lemma can be carried out in a similar manner.

Using the two lemmas above, one can prove the theorem. Since $\left\{f\left(t_{i}\right)\right\}$ and $\left\{g\left(t_{i}\right)\right\}$ are inverse permutations, there are exactly three cases to eliminate: (1) up-crossing at $t_{k}$ and down-crossing at $f\left(t_{k}\right)$, (2) up-crossing at $t_{k}$ and touching at $f\left(t_{k}\right),(3)$ down-crossing at $t_{k}$ and touching at $f\left(t_{k}\right)$. We remark that if there is an up-crossing at $t_{k}$, then $f\left(t_{k}\right)=t_{m}$ must lie between $f\left(t_{k-1}\right)$ and $f\left(t_{k+1}\right)$. Because, if this were not the case, $f\left(I_{k-1}\right)$ and $f\left(I_{k}\right)$ would have an interval $I_{m-1}$ or $I_{m}$ in common. But $g f\left(I_{k-1}\right) \subset\left[0, t_{k}\right]$ and $g f\left(I_{k+1}\right) \subset\left[t_{k}, 1\right]$, so that $g f\left(I_{k-1}\right)$ and $g f\left(I_{k}\right)$ cannot have an interval $g\left(I_{m-1}\right)$ or $g\left(I_{m}\right)$ in common. An immediate consequence is that $f\left(t_{k}\right) \neq 0,1$ if there is an up-crossing at $t_{k}$. In fact, $f\left(t_{k}\right) \neq t_{1}, t_{n}$. Also, we note that if there is a down-crossing at $t_{k}$ for which $f\left(t_{k}\right)=t_{m}$ does not lie between $f\left(t_{k-1}\right)$ and $f\left(t_{k+1}\right)$, then either $t_{m-1}$ or $t_{m+1}$ occurs as a value of $f\left(t_{k-1}\right)$ or $f\left(t_{k+1}\right)$. For example, if both $f\left(t_{k-1}\right)$ and $f\left(t_{k+1}\right)$ were greater than $t_{m}$, then one of them would be equal to $t_{m+1}$. This follows by applying Lemma 1 to the relations $t_{m+1}=f\left(t_{j}\right) \in f\left(I_{k-1}\right), t_{m+1}=f\left(t_{j}\right) \in f\left(I_{k}\right)$, to find $t_{k-1} \leqq t_{j} \leqq t_{k+1}$.

Case 1. Up-crossing at $t_{k}$ and down-crossing at $t_{m}=f\left(t_{k}\right)$. In this case $t_{m} \neq t_{1}, t_{n}$, and $I_{k-1}$ and $I_{m}$ are down-intervals while $I_{k}$ and $I_{m-1}$ are up-intervals. We will now show that neither $f\left(t_{k-1}\right)>t_{m}$ nor $f\left(t_{k+1}\right)>t_{m}$, contradicting the statement about up-crossings in the previous paragraph. If $f\left(t_{k-1}\right)>t_{m}$, then $f\left(I_{k-1}\right) \supset I_{m}$. Since $I_{k-1}$ is a down-interval,

$$
\left[0, t_{k}\right] \supset g f\left(I_{k-1}\right) \supset g\left(I_{m}\right),
$$

which implies that $g\left(I_{m}\right) \supset I_{k-1}$. This means $g f\left(t_{k-1}\right)=t_{k-1} \in g\left(I_{m}\right)$, which by Lemma 1 implies $f\left(t_{k-1}\right) \leqq t_{m+1}\left(I_{m}\right.$ is a down-interval). This leaves only the possibility $f\left(t_{k-1}\right)=t_{m+1}$, which by Lemma 2 implies 
$I_{k-1}$ and $I_{m}$ are not alike. Contradiction. If on the other hand $f\left(t_{k+1}\right)$ $>t_{m}$, then $f\left(I_{k}\right) \supset I_{m}$. Since $I_{k}$ is an up-interval,

$$
\left[t_{k}, 1\right] \supset g f\left(I_{k}\right) \supset g\left(I_{m}\right),
$$

which implies $g\left(I_{m}\right) \supset I_{k}$. This means $g f\left(t_{k+1}\right)=t_{k+1} \in g\left(I_{m}\right)$, which by Lemma 1 implies $f\left(t_{k+1}\right) \leqq t_{m+1}$. This leaves only the possibility $f\left(t_{k+1}\right)$ $=t_{m+1}$, which by Lemma 2 implies $I_{k}$ and $I_{m}$ are alike. Contradiction.

Case 2. Up-crossing at $t_{k}$ and touching at $t_{m}=f\left(t_{k}\right)$. Once again $t_{m} \neq t_{1}, t_{n}$. In the proof under Case 1 , we showed that neither $f\left(t_{k-1}\right)$ $>t_{m}$ nor $f\left(t_{k+1}\right)>t_{m}$ if $I_{m}$ is a down-interval. By a completely analogous proof, we can show that neither $f\left(t_{k-1}\right)<t_{m}$ nor $f\left(t_{k+1}\right)<t_{m}$ if $I_{m-1}$ is an up-interval. In case of a touching at $t_{m}, I_{m-1}$ and $I_{m}$ are alike, implying that either $I_{m}$ is a down-interval or $I_{m-1}$ is an up-interval. Thus, $t_{m}$ cannot lie between $f\left(t_{k-1}\right)$ and $f\left(t_{k+1}\right)$, giving a contradiction.

Case 3. Down-crossing at $t_{k}$ and touching at $t_{m}=f\left(t_{k}\right)$. Suppose first that $f\left(t_{k-1}\right)=t_{m+1}$. Lemma 2 implies that $I_{m}$ is a down-interval. This means $t_{m} \neq t_{1}$ and that $I_{m-1}$ is also a down-interval. Now, $g\left(I_{m-1}\right)$ contains either $I_{k-1}$ or $I_{k}$, so that either $g f\left(t_{k-1}\right)=t_{k-1} \in g\left(I_{m-1}\right)$ or $g f\left(t_{k+1}\right)$ $=t_{k+1} \in g\left(I_{m-1}\right)$. By Lemma 1 then, either $f\left(t_{k-1}\right) \leqq t_{m}$ or $f\left(t_{k+1}\right) \leqq t_{m}$. The only possibility is $f\left(t_{k+1}\right)<t_{m}$. This in turn means $f\left(I_{k}\right) \supset I_{m-1}$, so that

$$
\left[0, t_{k+1}\right] \supset g f\left(I_{k}\right) \supset g\left(I_{m-1}\right) .
$$

Thus, either $g\left(t_{m-1}\right)=t_{k+1}$ or $g\left(I_{m-1}\right) \supset I_{k-1}$. The case $g\left(t_{m-1}\right)=t_{k+1}$ is eliminated by Lemma 2 which would imply that $I_{m-1}$ and $I_{k}$ are not alike, a contradiction. On the other hand, $g\left(I_{m-1}\right) \supset I_{k-1}$ implies $g f\left(t_{k-1}\right)=t_{k-1} \in g\left(I_{m-1}\right)$ which by Lemma 1 gives $f\left(t_{k-1}\right) \leqq t_{m}$, a contradiction. The cases $f\left(t_{k-1}\right)=t_{m-1}, f\left(t_{k+1}\right)=t_{m-1}, f\left(t_{k+1}\right)=t_{m+1}$ can be eliminated in a similar manner. (It is left to the reader to show that the cases $t_{k}=t_{1}, t_{n}$ have been eliminated by the previous argument.) According to the remark preceding the discussion of Case 1, we have only to consider the case in which $t_{m-1}, t_{m}$, and $t_{m+1}$ all lie between $f\left(t_{k-1}\right)$ and $f\left(t_{k+1}\right)$. Suppose $f\left(t_{k-1}\right)<t_{m-1}, t_{m}, t_{m+1}<f\left(t_{k+1}\right)$. Then, $f\left(I_{k-1}\right) \supset I_{m-1}$ and $f\left(I_{k}\right) \supset I_{m}$. But

$$
\begin{aligned}
& {\left[t_{k-1}, 1\right] \supset g f\left(I_{k-1}\right) \supset g\left(I_{m-1}\right),} \\
& {\left[0, t_{k+1}\right] \supset g f\left(I_{k}\right) \supset g\left(I_{m}\right) .}
\end{aligned}
$$

Therefore, $g\left(I_{m-1}\right) \supset I_{k}$ and $g\left(I_{m}\right) \supset I_{k-1}$. This means that $g f\left(t_{k+1}\right)$ $=t_{k+1} \in g\left(I_{m-1}\right)$ and $g f\left(t_{k-1}\right)=t_{k-1} \in g\left(I_{m}\right)$. Since $I_{m}$ and $I_{m-1}$ are alike, Lemma 1 states that either $f\left(t_{k+1}\right) \leqq t_{m}$ or $f\left(t_{k-1}\right) \geqq t_{m}$. Contradiction. 
The remaining case in which $f\left(t_{k+1}\right)<t_{m-1}, t_{m}, t_{m+1}<f\left(t_{k-1}\right)$ is handled in a similar fashion.

As a final remark, we note that in the proofs there was no essential use made of the commutativity property of $f(t)$ and $g(t)$ except at fixed points of $h(t)$.

\section{REFERENCE}

1. J. R. Isbell, Commuting mappings of trees, Research Problem 7, Bull. Amer. Math. Soc. 63 (1957), 419.

UNIVERSITY OF MiNNESOTA 